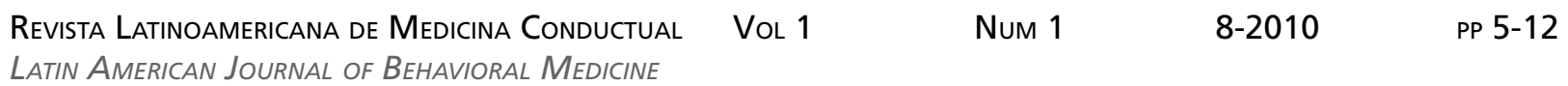

Graciela Rodríguez Ortega ${ }^{1}$

Facultad de Psicología, Universidad Nacional Autónoma de México

Recibido: Mayo 31, 2010

Revisado: Junio 26, 2010

Aceptado: Junio 27, 2010

\section{Desarrollo de la Medicina Conductual en MÉXICO}

\author{
Development of Behavioral Medicine in \\ MEXICO
}

Dirigir correspondencia a: Edificio "D", piso 3, cubículo de Medicina Conductual, Facultad de Psicología, UNAM, Ave. Ciudad Universitaria 3000, Col. Coyoacán Universidad, Delegación Coyoacán, México, D. F., C. P. 04360.

Correo: rodrigue@servidor.unam.mx

\section{Resumen}

Este artículo describe el paralelismo de México con respecto a otros países en relación a los factores que determinaron el surgimiento de la Medicina Conductual, entre ellos se desatacan a la biorretroalimentación, el análisis conductual aplicado y el cambio en el perfil epidemiológico. Como campo interdisciplinario, la medicina conductual, integra conocimientos de la ciencia conductual con la biomédica dando como resultado la conjunción de diversos saberes. En México, se han estudiado interdisciplinariamente diversas problemáticas como las enfermedades crónicas degenerativas, cardiovasculares, diabetes, cáncer, entre otras. Sin embargo, dado los cambios en la organización política, social y económica del país se hace necesaria la incursión en áreas como políticas públicas, derechos humanos y bioética.

Palabras clave: Medicina conductual, análisis conductual aplicado, psicología de la salud, interdisciplina y desarrollo.

\section{Abstract}

This article describes the parallelism of Mexico with respect to other countries regarding the factors that determined the emergence of Behavioral Medicine, among them are biofeedback, applied behavior analysis and change in the epidemiological profile. As an interdisciplinary field, behavioral medicine, integrates the behavioral sciences, biomedical sciences and social science resulting in the conjunction of different knowledge. In Mexico, we have studied various interdisciplinary problems such as chronic degenerative diseases, cardiovascular diseases, diabetes, cancer, among others. However, given the changes in the political, social and economic organizations of our country it becomes necessary to venture into areas such as public policies, human rights and bioethics.

Keywords: Behavioral medicine, applied behavioral analysis, health psychology, interdisciplinary and development. 
El presente artículo tiene como objetivos mostrar determinados acontecimientos históricos que dieron origen al surgimiento de la Medicina Conductual en México, además analiza aspectos relacionados con los conceptos y paradigmas que dan origen a la evidencia empírica en esta área, así como describir algunos avances en nuestro país acerca de los problemas de salud que corresponden al campo de la Medicina Conductual.

\section{Acontecimientos históricos}

En 1977 se organiza el Primer Congreso sobre Medicina Conductual en la Universidad de Yale. Se reunieron importantes especialistas del área de la salud y de las ciencias sociales para formalizar la creación de la medicina conductual, entendida como un campo interdisciplinario de integración de conocimientos biomédicos y sociales, con el fin de diseñar e implementar procedimientos para la prevención, el tratamiento y la rehabilitación de pacientes, siendo uno de sus principales resultados la definición más específica del campo de aplicación de la medicina conductual.

En 1978 se funda la Academia de Investigación en Medicina Conductual, en Estados Unidos, ésta define a la medicina conductual como el campo interdisciplinario que se ocupa de la integración de la ciencia de la conducta con la biomédica, con el fin de desarrollar y aplicar los conocimientos y técnicas dirigidas a la promoción de la salud; prevención, diagnóstico, tratamiento y rehabilitación de las enfermedades (Schwartz \& Weiss, 1978).

A partir de entonces, el campo de la medicina conductual comenzó a crecer rápidamente, se publicó el primer número del Journal of Behavioral Medicine en marzo de 1978. Su primer editor fue W. Doyle Gentry, profesor de psicología médica de la Universidad de Duke. Posteriormente se publicó la Revista Biofeedback and Self-Regulation, en donde se reportaban investigaciones sobre la aplicación de las técnicas y procedimientos basados en la medicina conductual a enfermedades, tales como hipertensión arterial, dolor crónico, asma infantil, desórdenes cardiovasculares, rehabilitación neuromuscular, y cáncer.

Asimismo, se estableció la Academy Research in Behavioral Medicine en Estados Unidos, cuyo primer presidente fue el psicólogo Neal Miller. Además, se integró como miembro permanente de la Study Section on Behavioral Medicine, perteneciente al National Institute of Health; y desde entonces es miembro activo del National Heart, Lung and Blood Institute y del National Cancer Institute.
En la década de los años 70s, las principales aportaciones a la medicina conductual se hicieron desde el área del análisis experimental de la conducta. Fueron muy relevantes las aplicaciones clínicas del biofeedback, así como los intentos de extender los principios del condicionamiento clásico y del condicionamiento operante al tratamiento de múltiples enfermedades y/o padecimientos.

Mientras que Pomerlau y Brady (1979) propusieron como características inherentes a la medicina conductual: a) el uso de técnicas clínicas surgidas del análisis experimental de la conducta, que se enmarcaban bajo los rubros genéricos de Terapia y Modificación de la conducta; y b) una tendencia hacia la investigación, teniendo como soporte metodológico el análisis funcional de las conductas asociadas a las enfermedades y desórdenes médicos.

Blanchard (1982), al referirse a la historia de la medicina conductual, menciona tres acontecimientos que favorecieron el surgimiento de la misma en la década de los años 70's. Éstos fueron: a) Utilización de un conjunto de técnicas efectivas para modificar el comportamiento y que habían sido utilizadas con éxito en enfermos mentales, pero que comenzaban a ser aplicadas a pacientes con otros problemas de salud (obesidad, tabaquismo, adicciones, entre otros). b) El desarrollo del campo de la biorretroalimentación estaba demostrando que era posible efectuar cambios fisiológicos en funciones corporales, reguladas por el sistema nervioso autónomo, abriendo la posibilidad de realizar intervenciones terapéuticas efectivas para trastornos somáticos (migraña, hipertensión, cefaleas de tensión, úlceras y dolor crónico). c) Se dirigió la atención hacia las dos principales causas de mortalidad en adultos, cáncer y enfermedades cardiovasculares, además de que se reconocía el papel importante que los cambios en el comportamiento jugaban en el tratamiento y prevención de esas enfermedades.

Por otra parte, Agras (1982) señala cuatro factores que favorecieron el rápido crecimiento de la medicina conductual. a) El antecedente de que la medicina psicosomática había generado un cuerpo de investigación que vinculaba los factores psicológicos, a un número importante de enfermedades. b) El análisis conductual aplicado, como área de intervención, había generado procedimientos de tratamiento psicológico, conductualmente orientados a una variedad de trastornos físicos. c) Los estudios epidemiológicos habían identificado ciertos factores que incrementaban el riesgo asociado con algunas enfermedades. d) Como consecuencia del aumento en los costos del 
tratamiento de las enfermedades, se produjo interés por la prevención en lo general, y en particular, en los factores psicológicos y sociológicos involucrados en el trabajo preventivo.

\section{Aspectos conceptuales}

El campo de la medicina conductual surgió básicamente por iniciativa de los psicólogos que trabajaban en el campo de la salud, pero los profesionales médicos sintonizaron de inmediato con el nuevo enfoque dado su carácter científico y su aplicación en diversos escenarios. De tal forma que, en 1984, Orth-Gomer describe sus distintos niveles de interacción: a) Entre disciplinas, en la cual se reconoce que las ciencias del comportamiento pueden ser enlazadas con el conocimiento de las ciencias biológicas, por ejemplo, el impacto de las medidas de personalidad en la predicción de enfermedades cardiovasculares. b) Entre factores de riesgo, donde se vinculan los factores que incrementan el riesgo de contraer una enfermedad o de morir de ella (factores de riesgo médicos y conductuales). c) Entre conceptos, demostrado cuando una teoría se enriquece al integrar conceptos originados en distintos campos del conocimiento. d) De profesionales, relacionado al diálogo entre científicos con diferente formación académica y cultural para la interacción de las disciplinas.

Agras (1982) ha señalado que el desarrollo de la medicina conductual implica una aproximación científica diferente al campo de la Salud-Enfermedad, lo cual conduce a llevar a cabo actividades de investigación interdisciplinaria. Bajo esta aproximación se reconoce que el conocimiento y experiencia de diversas disciplinas son necesarias para comprender e intervenir en la naturaleza multifacética de los problemas de salud actuales.

Considerando la gran variedad de disciplinas que contribuyen a los problemas de la salud y la enfermedad, el concepto de Medicina Conductual ofrece el espacio de discusión interdisciplinaria para intercambiar ideas y enfoques teóricos al respecto. El elemento principal de este paradigma es que no es necesario que todas las disciplinas tengan una misma perspectiva para que resulte efectiva la colaboración entre ellas. Lo anterior sustenta el cambio del concepto multidisciplina a interdisciplina, lo cual sólo puede ser llevado a cabo cuando todos los participantes reconocen el valor que representan otras disciplinas al aportar diferentes perspectivas y formas de solución ante de problemáticas de salud.

Como parte del modelo de la Medicina Conductual es relevante describir los conceptos de interac- ción y variabilidad. Interacción se refiere a la conjunción de disciplinas, niveles de análisis, así como el efecto de la combinación de variables. Mientras que la variabilidad se refiere a la evaluación del rango de responsividad fisiológica a una amplia gama de problemas ambientales. Por ejemplo, las elevaciones de la presión arterial y la frecuencia cardiaca muestran un índice de reactividad cardiovascular a los cambios físicos y/o psicológicos. Si las personas con perfiles de hiperreactividad están en riesgo de padecer enfermedades cardiovasculares la medición del perfil permitiría analizar e intervenir en este caso (Weiss \& Miller, 1999). Este es sólo un ejemplo que permite comprender la intervención conjunta e interdisciplinaria con otros profesionales de la salud orientados a prevenir disfunciones orgánicas y/o enfermedades.

La aproximación bioconductual que plantea la medicina conductual sugiere un complejo escenario de interacción de múltiples variables (ambientales, psicológicas, fisiológicas, sociológicas y nutricionales) en diferentes niveles, dando como resultado variaciones en las respuestas fisiológicas, de tal forma que un cambio en determinada variable podría determinar una disfunción orgánica. De esta manera se ha abordado la relación entre el impacto psicológico y el estatus fisiológico de determinados enfermedades.

El campo de la medicina conductual aborda el trabajo conjunto, integral y complementario hacia la atención de pacientes con problemas crónicos, entre otros, a través de diferentes modelos de intervención, que implican básicamente entrenar al paciente para aprender a vivir en mejores condiciones y evitar deterioros psicológicos y conductuales para mejorar la calidad de vida del paciente. Los modelos clínicos que han demostrado mayor eficacia al abordar problemáticas de salud son los derivados de la Teoría Cognitivo Conductual (Martínez y Piqueras, 2010).

\section{Desarrollo de la Medicina Conductual en México}

A través del trabajo e investigaciones, los expertos en medicina conductual en México han demostrado la importancia de diversos componentes psicológicos en las enfermedades, así como su interrelación con factores medioambientales. Como resultante, el papel del psicólogo se ha expandido enormemente y se comprueba que los estilos de vida, el vivir en áreas altamente contaminadas, el sobrepeso, la vida sedentaria, utilizar cinturones de seguridad, fumar, consumir alcohol en exceso, y el estrés cotidiano, influyen en nuestra salud, y que los tratamientos únicamente médicos, no reditúan los beneficios necesarios hacia nuestra salud; por lo tanto, es necesario identificar 
conductas que reduzcan el riesgo de padecer ciertas enfermedades; y favorecer conductas saludables, lo que implica un cambio y mantenimiento de un nuevo estilo de vida.

La medicina conductual incorpora las aportaciones científicas, tecnológicas y profesionales de otros campos, pues reconoce que las aportaciones de diversas disciplinas, es necesaria para comprender la naturaleza multifacética de los problemas de salud en el mundo contemporáneo, demostrado así su éxito (Anderson, 1999; Blanchard, 1992; Rodríguez y Rojas, 1998).

Ahora bien, hablar del desarrollo de la medicina conductual en México, implica hacer cierto paralelismo con lo que ocurrió en Estados Unidos. Por ejemplo, a principios de los años 60s, Víctor Alcaraz, Emilio Ribes, Antonio Gago, Florente López y Serafín Mercado marcaron el inicio del análisis de la conducta en México cuando se encontraban en Universidad Veracruzana (Xalapa, Ver.) dando el impulso de una psicología científica basada en el análisis experimental de la conducta. En 1966 se incorporó otra importante generación de analistas conductuales encabezados por Gustavo Fernández Pardo, Javier Aguilar Villalobos y Arturo Bouzas (Martínez, 2006). En 1967 Florente López y Francisco Barrera crearon el Centro de Entrenamiento y Educación Especial, que constituiría el primer centro de análisis conductual aplicado en México (Martínez, 2006).

En 1975 se celebró en la Ciudad de México, el Tercer Congreso Latinoamericano de Análisis de la Conducta, con la destacada participación de B. F. Skinner. Todas estas circunstancias se conjuntaron para crear las condiciones que sentaron las bases para la consolidación de la investigación conductual en México.

Entre tanto, Benjamín Domínguez incursionó en diversos reclusorios (para adultos, jóvenes y niños), consiguiendo modificar algunas de las prácticas perniciosas que se daban en estos ambientes (Domínguez, Rueda, Maklouff y Rivera, 1975). También en algunos hospitales psiquiátricos incursionaron diversos analistas conductuales quienes consiguieron aplicar técnicas y terapias alternativas (modificación de conducta), diferentes a las utilizadas en estos ámbitos (evaluación psicométrica y aplicación de fármacos). Tal fue el caso del Hospital Psiquiátrico Infantil Juan N. Navarro (SSA).

Otros escenarios importantes de aplicación fueron los estudios llevados a cabo sobre el desarrollo infantil. Analistas conductuales dirigidos por Héctor Ayala dieron origen a programas basados en el análisis conductual aplicado en las áreas de retardo en el desarrollo; tratamiento y rehabilitación de pacientes mentales crónicos; y con niños y adolescentes con problemas de consumo de drogas (Ayala, Quiroga, Mata \& Chism, 1981; Ayala, Chism, Cárdenas, Rodríguez, Cervantes \& Caballero, 1982), en los cuales se trabajó interdisciplinariamente con otros profesionales de la salud.

Importantes intervenciones realizadas en el campo de la medicina conductual en México fueron las llevadas a cabo por Xóchitl Gallegos y Víctor Alcaraz en los años 80's, quienes aplicaron el biofeedback para el tratamiento de migrañas, parálisis facial, arritmias cardíacas, control de esfínteres y ciertas paresias y tipos de epilepsia (Alcaraz, 1977; Alcaraz, CastroVelázquez, De la Cruz \& Del Valle, 1981; Gallegos \& Torres Torrija, 1983; Gallegos, Medina, Espinoza \& Bustamante, 1987).

En el Laboratorio de Plasticidad Cerebral, coordinado por Jorge Palacios y fundado en 1984 dentro de la Facultad de Psicología de la UNAM, se utilizó también la retroalimentación para el tratamiento de diversas parálisis neuromusculares con gran éxito.

En la Escuela Nacional de Estudios Superiores Zaragoza (UNAM) se conformó un grupo de trabajo encabezado por Luis Florez Alarcón, que tuvo como objetivo la creación de una línea de investigación sobre las aplicaciones clínicas del condicionamiento instrumental de respuestas autónomas. Posteriormente este grupo de trabajo dio origen al Programa de Psicología de la Salud conjuntamente con la Secretaria de Salud y la Facultad de Psicología, que se estableció desde 1984 hasta 1999. Este programa se vio enriquecido con la participación de Víctor Alcaraz y Ana Eugenia Díaz de León, procedentes de la Facultad de Psicología, quienes desarrollaron de manera colaborativa el primer proyecto de investigación sobre el condicionamiento de ritmos eléctricos cerebrales para el tratamiento de la epilepsia (Rojas, 2002).

Durante la segunda mitad de los ochentas, el programa sufre una transformación gradual y significativa, quedando al frente Mario Rojas Russell quien realiza investigaciones sobre Diabetes mellitus y peso corporal como factores de riesgo (Anaya, Carvajal, Charles, Hurtado, Mayor, Rojas y Sánchez, 2002), así como elaboración de documentos relevantes sobre la psicología clínica y de la salud (García, Rojas, Vargas, 2001; Rojas, 2002; 2005). Es relevante mencionar que actualmente en esta Facultad de Zaragoza se ha creado la Unidad de Cardiología Conductual en donde se realizan investigaciones interdisciplinarias sobre los factores psicosociales 
que favorecen la aparición y el desarrollo de enfermedades cardiovasculares (Figueroa, Domínguez, Ramos del Rio, 2009).

En la Escuela Nacional de Estudios Superiores Iztacala (UNAM), Rocío Hernández Pozo fundó el Laboratorio de Conducta Humana Compleja, iniciando proyectos de investigación relacionados con la evaluación de factores de riesgo cardiaco con sistemas computarizados (Rodríguez, Hernández-Pozo, Álvarez y Negrete, 1989); sobre la creación de un sistema experto computarizado de evaluación (Rodríguez, Hernández-Pozo, Fernández y Negrete, 1992); programa de cómputo para evaluar la tolerancia a la frustración en atletas mexicanos (Hernández-Pozo, Serrano, Méndez, Montes y Rodríguez, 1992); así como la enseñanza de comportamiento saludable vía escenarios virtuales (Hernández-Pozo, Harzem y Rossi, 1990; Hernández-Pozo, 1998).

De forma paralela, en la Facultad de Psicología de la UNAM, el grupo de trabajo coordinado por Graciela Rodríguez realizaron los primeros estudios sobre trasplantes de órganos y tejidos en población mexicana (Rodríguez, Pérez, Rojas, Reyes y Méndez, 2004). Además, Benjamín Domínguez comenzó a trabajar sobre el manejo del dolor crónico (Domínguez, 1995; 1999, 2000), en el área de la psiconeuroinmunología (Domínguez, 2000, 2001); así como en la retroalimentación biológica (Domínguez, 2000).

En el área de la psicología ambiental con enfoque en la medicina conductual se han destacado los trabajos de Serafín Mercado, Patricia Ortega y Javier Urbina. En esta área se han trabajado aspectos relacionados con el estrés ambiental en instituciones de salud (Ortega, 2004, 2005), sobre el impacto ambiental de los escenarios hospitalarios sobre los pacientes y sus familiares, así como con médicos y enfermeras (Ortega, y Urbina, 1984; López, Ortega y Acuña, 1990; Ortega y Aguilar, 2003).

En resumen, en un estudio llevado a cabo en México se pudieron identificar las contribuciones científicas, tecnológicas y de intervención de equipos interdisciplinarios, encontrándose que las líneas de investigación giran en torno al desarrollo de modelos de intervención, sobre epidemiología conductual, educación para la salud, salud y género, envejecimiento normal y patológico, trasplante de órganos y tejidos, adherencia terapéutica, conducta agresiva y comportamiento antisocial, diabetes, cáncer, enfermedades cardiovasculares, VIH/SIDA, adicciones, estrés, dolor crónico, ira, hostilidad, biofeedback, políticas públicas, derechos humanos y bioética (Rodríguez y Rojas, 1998; Rodríguez y Jaén, 2004).
Como puede observarse la Medicina Conductual en México, se ha caracterizado por reunir científicos de diferentes campos de conocimiento con el objetivo de definir acciones que puedan permitir acceder a la mejor calidad de vida a la población mexicana y así, enfrentar los principales retos de la atención a la salud.

\section{Difusión de la Medicina Conductual}

De esta manera en el 2001, se funda la Sociedad Mexicana de Medicina Conductual, siendo su presidenta fundadora Graciela Rodríguez Ortega, posteriormente Javier Urbina Soria y actualmente es José Luis Ibarrola Calleja. En el 2002 se organiza el Coloquio de Medicina Conductual con la participación de la Facultad de Psicología y la Asociación para Evitar la Ceguera en México. En el 2005, junto con la Sociedad Internacional de Medicina Conductual, se organiza la Primera Reunión Regional Latinoamericana y del Caribe de Medicina Conductual en la Ciudad de México. En el 2006 se lleva a cabo el Coloquio de Medicina Conductual "Manejo de las enfermedades crónicodegenerativas y emergentes: una visión hacia el futuro". Finalmente, en el 2009 se organiza Coloquio de Medicina Conductual en donde participaron científicos de otras entidades de la República (Veracruz, Guadalajara, San Luis Potosí, Chihuahua, Cuernavaca), así como de otras disciplinas relacionadas a la salud. De esta manera se reconoció el desarrollo de la medicina conductual en diversas partes de México, así como el trabajo en diferentes escenarios, tanto urbanos como rurales. Es importante mencionar que como resultado del primer Coloquio de Medicina Conductual se editó el primer libro de Medicina Conductual en México.

Paralelamente, en los diversos congresos de psicología en el país han sido recurrentes las presentaciones de trabajos y conferencias sobre temas de salud y comportamiento. Lo cual ha permitido configurar líneas, temas y niveles de intervención o participación de los profesionales de la salud en el ámbito de la cardiología, neumología, oftalmología y otras especialidades médicas.

Lo anterior resulta relevante si consideramos que la American Psychological Association (2000), determinó que el periodo comprendido entre el 2000-2010 es la "Década del Comportamiento", cuya premisa indica que los principales problemas que aquejan a la población mundial pueden prevenirse o mitigarse mediante cambios conductuales, centrando sus metas en mejorar la educación y la atención a la salud, aumentar la seguridad en hogares y comunidades, y atender las necesidades de una población que enve- 
jece, destacando el papel de la Medicina Conductual en este sentido.

Sin embargo, existen retos que aún tenemos que enfrentar en México. Por ejemplo, impulsar la investigación en este campo no sólo en las instituciones educativas sino también en las instituciones del sector salud y/o social. Asimismo, impulsar la conformación de estudios de posgrado que permitan formar cuadros de profesionales así como abarcar diversas especialidades en relación al cada vez más complejo estado de salud de la sociedad. Además, establecer mecanismos que permitan identificar y reunir a todos aquellos profesionales del resto de las ciencias de la salud que llevan a cabo investigación, formación de recursos humanos y trabajo profesional en Medicina Conductual, de modo tal que exista congruencia con el espíritu inter, multidisciplinario y transdiciplinario de este campo de estudio.

\section{Agradecimientos}

Quiero reconocer a Rocío Hernández Pozo por su brillante desarrollo y el haberme introducido dentro de esta área del conocimiento, en la cual hemos compartido foros internacionales.

Agradezco el valioso trabajo de Ángeles Mata, que con su conocimiento del desarrollo de la psicología en México ha sido posible recuperar pinceladas de nuestra historia.

Asimismo, quiero agradecer a Alejandra Cruz Cruz por todo el interés mostrado acerca de los aspectos conceptuales de la medicina conductual, además de la recopilación de información necesaria para el mismo.

Finalmente, doy gracias a todas aquellas personas que tuvieron a bien enviarme los nombres de sus líneas de investigación en el área de la medicina conductual, fueron valiosos para este trabajo, que aunque incompleto marcan el camino hacia el rumbo de la Medicina Conductual en México.

\section{Referencias}

Agras, W. S. (1982). Behavioral Medicine in the 1980s: Nonrandom connections. Journal of consulting and clinical psychology, 50 (6), 797-803.

Alcaraz, R. V. (1977). Extensiones de las técnicas de condicionamiento a las perturbaciones psicosomáticas y a la medicina. Enseñanza e Investigación en Psicología, 3, 83-96.

Alcaraz, R. V., Castro-Velázquez, I., De la Cruz, S., \& Del Valle, G. (1981). Conditioning and recovery of functions lost by brain damage. Neurocience and Biobehavioral Reviews, 5, 371-383.
American Psychological Association (2000). Decade of Behavior. Disponible en http://www.decadeofbehvaior.org

Anaya, R., Carvajal, N., Charles, G., Hurtado, M., Mayor, H., Rojas, M., \& Sánchez, L. (2002). Diabetes mellitus y peso corporal como factores de riesgo para desarrollar cáncer de endometrio: Un estudio de casos y controles. Archivos en Medicina Familiar, 4 (4), 121-126.

Anderson, N., B. (1999). An assessment, a report card and a prescription for actualizing our potential. Conferencia en la Reunión Anual 1999 de la Society of Behavioral Medicine.

Ayala, V. H., Chism, S. K., Cárdenas, L. G., Rodríguez, C. M., Cervantes, L., \& Caballero, F. P. (1982). Una alternativa al tratamiento y rehabilitación del paciente mental crónico hospitalizado, Salud Mental, 5, 87-93.

Ayala, V. H., Quiroga, A. H., Mata, M. A., \& Chism, S. K. (1981). La familia enseñante: evaluación del modelo en México en términos de reincidencia en su aplicación en una muestra de niños inhaladores de solventes industriales. Salud Mental, 4, 11-15.

Blanchard, E. B. (1982). Behavioral Medicine: Past, present and future. Journal of consulting and clinical psychology, 50, 795-796.

Blanchard, E. B. (1992). Introduction to the special issue on Behavioral Medicine: An update for the 1990s. Journal of Consulting and Clinical Psychology, 60, 491-492.

Domínguez, B., Rueda, M., Maklouff, C., \& Rivera, A. (1975). El análisis y el control de las actividades de grupos humanos bajo custodia. En: A. Bandura y E. Ribes (Eds.), Modificación de conducta: Análisis de la agresión y la delincuencia. México: Trillas.

Domínguez, T. B. (1995). Investigación Psicológica y Manejo No-Invasivo del Dolor Crónico. Psicología Contemporánea, 2 (1), 64-75.

Domínguez, T. B. (1999). Therapeutic Potential of Emotional Disclosure for Chronic Pain Relief. A Mexican Experience. En Abstracts 9th World Congress of Pain, August, 22-27, Vienna, Austria.

Domínguez, T. B. (2000). Autorregulación del dolor crónico. En L. Oblitas y C. Becoña (Comps.) Psicología de la Salud (pp. 179-197). México: Plaza y Valdés.

Domínguez, T. B. (2000). Psicoinmunología: Procesos Psicológicos, Inmunosupresión y Efectos en la Salud. Psicología y Salud, 10 (2), 31-45. 
Domínguez, T. B. (2000). Retroalimentación Biológica Sustentable y Nacional, ¿Por Qué? ¿Para Quién? Enseñanza e Investigación en Psicología, 5 (2), 359-371.

Domínguez, T. B. (2001). Psiconeuroinmunología. Procesos Psicológicos, Inmunosupresión y Efectos en la Salud. Ciencia y Desarrollo, 27 (159), 28-35.

Figueroa C., Domínguez, B., Ramos del Rio, B., \& Alcocer, L. (2009). Emergencia de la cardiología conductual en la práctica clínica. Psicología y Salud, 19 (1), 151-155.

Gallegos, X., \& Torres-Torrija, J. (1983). Biorretroalimentación de la respuesta galvánica de la piel y entrenamiento en relajación: un estudio piloto. Revista Latinoamericana de Psicología, 15, 259-275.

Gallegos, X., Medina, R., Espinoza, E., \& Bustamante, A. (1987). Retroalimentación biológica en la rehabilitación de parálisis facial bilateral: reporte de un caso. Trabajo presentado en el XXI congreso Interamericano de Psicología. Habana, Cuba.

García, M., Rojas, M., \& Vargas, I. (2001). Psicología clínica y salud: Perspectivas teóricas. México, Distrito Federal: Universidad Nacional Autónoma de México.

Hernández-Pozo, M. R. (1998) Empleo de pruebas ejecución computarizadas para la evaluación de estilos conductuales, conducta inteligente y factores de riesgo para problemas de salud. En V. Alcaraz y A. Bouzas (Eds.) Las aportaciones mexicanas a la Psicología: La perspectiva de la investigación (pp. 771-787). México: UNAM.

Hernández-Pozo, M. R., Harzem, P., \& Rossi M. (1990). Ejecución de adultos bajo el paradigma de autocontrol en un videojuego computarizado. Revista Mexicana de Análisis de la Conducta, 16, (3), 71-85.

Hernández-Pozo, M. R., Serrano, A., Méndez, J., Montes, E., \& Rodríguez, G. (1992) Computerized operant evaluation of tolerance to frustration in high performance mexican athletes and marathon runners. Proceedings of the International Conference on Computer Applications in Sport and Physical Education. Netanya: Wingate Institute.

López, E., Ortega, P., \& Acuña, M. (1990). Efectos de dos diseños de oficina en la comunicación, en la percepción del ambiente de trabajo y en las respuestas emocionales de las personas. En Asociación Mexicana de Psicología (Ed.) La Psi- cología Social en México (pp. 178-182). MéxicO: AMEPSO.

Martínez, S. H. (2006). Treinta años de la Revista Mexicana de Análisis de la Conducta: Un reto a la supervivencia. Avances en Psicología Latinoamericana, 24, 105-125.

Martínez-González, A. E., \& Piqueras-Rodríguez, J. A. (2010). Eficacia de la terapia cognitivo-conductual en trastornos afectivos y de ansiedad mediante neuroimagen funcional. Revista de Neurología, 50 (3), 167-178.

Ortega, P., \& Aguilar, J. (2003). Estrés ambiental: un modelo de salas de espera hospitalarias. Revista Mexicana de Psicología, 20 (2) 327-336.

Ortega, P., \& Urbina, J. (1984). Estudio experimental de los efectos de hacinamiento sobre la ejecución de tareas y la percepción de las características ambientales de un escenario laboral. Revista Mexicana de Psicología, 2, 128-133.

Ortega, P. (2004). Aportaciones de la Psicología Ambiental a la Medicina Conductual: el diseño ambiental de hospitales. En G. Rodríguez (Coord.) Medicina Conductual en México (399414). México: Miguel Ángel Porrúa y Cámara de Diputados, LIX Legislatura.

Ortega, P. (2005). Estrés ambiental en instituciones de salud: valoración psicoambiental. México: Facultad de Psicología, Universidad Nacional de México.

Orth-Gomer, K. (1984). The significance of interaction in Behavioral Medicine. Homeostasis, 35, 6-15.

Pomerleau, O. F., \& Brady, J. P. (1979). Behavioral Medicine: Theory and Practice. Baltimore: Williams and Wilkins.

Rodríguez, G., \& Jaén, C. (2004). Tendencias actuales de la investigación en Medicina Conductual en México. En G. Rodríguez (Coord.), Medicina Conductual en México (pp. 37-56). México: Sociedad Mexicana de Medicina Conductual, A.C.

Rodríguez, G., \& Rojas, M. (1998). La Psicología de la Salud en América Latina. México: Miguel Ángel Porrúa.

Rodríguez, G., Hernández-Pozo, M. R., Álvarez-ManiIla, J., \& Negrete, J. (1989). Computer program for a behaviourally based evaluation of cardiac risk in a Mexican population. En P. F. Lovibond y P. Wilson (Eds.). Clinical and Abnormal Psychology, Proceedings of the XXIV International Congress of Psychology (pp. 399-405). Amsterdam: Elsevier Science Publishers, B. V.

Rodríguez, G., Hernández-Pozo, M. R., Fernández, G., \& Negrete, J. (1992). Avances en Psicometría 
Operante: Análisis experimental de estilos de personalidad y creación de un sistema experto computarizado de evaluación. Investigación e Innovación Docente en Psicología. México: Facultad de Psicología, UNAM.

Rodríguez, G., Pérez, C., Rojas, M., Reyes, I., \& Méndez, I. (2004). Trasplante de órganos y tejidos: Creencias, intenciones y actitudes en la población mexicana. En: G. Rodríguez (Coord.). Medicina Conductual en México. México: Cámara de Diputados LIX Legislatura, Sociedad Mexicana de Medicina Conductual, Miguel Ángel Porrúa Editor.

Rojas Russell, M. (2002) Análisis del programa de Psicología de la Salud. En: M. García Méndez y M. R. Cuevas Martínez (Comps.) Autoevalua- ción de la Carrera de Psicología, (pp. 177-192). México D.F: FES Zaragoza-UNAM.

Rojas, M. E. (2005). Aspectos epidemiológicos y psicológicos de la actividad y la inactividad físicas. En: L. Flórez-Alarcón, M. Botero y B. Moreno (Eds.). Psicología de la Salud: Temas actuales de investigación en Latinoamérica. Bogotá, Colombia: ALAPSA-Barranquilla, Colombia: UNINORTE.

Schwartz, G. E., \& Weiss, S. M. (1978.). Yale Conference of Behavioral Medicine: A Proposed Definition and Statement of Goals. Journal of Behavioral Medicine, 1 (1), 3-12.

Weiss, S. M., \& Miller, N. E. (1999). Behavioral Medicine. En G. Adelman y B. Smith (Eds.). Encyclopedia of Neuroscience. EUA: Elsevier Science. 